\title{
L'efficacité des gestes mis en images
}

Par LUCIA ANGELINO

Université Libre de Bruxelles

\section{Introduction}

Parmi les symptômes de la crise de la modernité, on peut compter la perte des gestes expressifs et intentionnels (Gebärde) ${ }^{1}$, désormais devenus des gesticulations incohérentes et démesurées ou bien des gestes mécaniques (Geste), impossibles à enfermer dans un cadre signifiant et communicationnel. Pourtant, comme le souligne Giorgio Agamben dans ses Notes sur le geste $^{2}$, cette perte de la gestualité qui affecte notre société — dès la fin du $\mathrm{XIX}^{\mathrm{e}}$ siècle - a déclenché en même temps que des recherches scientifiques - centrées sur les troubles de la motricité -, une tentative précipitée de récupérer dans le domaine de l'esthétique la grâce des gestes perdus, qui parvient à son comble dans les arts performatifs ${ }^{3}$, la littérature et, enfin, de la

\footnotetext{
${ }^{1}$ La langue allemande permet de faire une distinction sémantique entre le geste intentionnel, le geste sous-tendu par l'intentionnalité (Gebärde) et le geste mécanique (Geste). Le geste sous-tendu par l'intentionnalité (Gebärde) prend tout son sens si l'on ajoute que la langue des signes, se dit en allemand « Gebärdensprache ». Sur ce thème, je me permets de renvoyer le lecteur à Lucia Angelino, Entre voir et tracer. Merleau-Ponty et le mouvement vécu dans l'expérience esthétique, Paris, Éditions Mimésis, 2014.

2 Giorgio Agamben, «Notes sur le geste », dans Id., Moyens sans fins, trad. fr. D. Valin, Paris, Éditions Payot \& Rivages, 1995, p. 68.

${ }^{3}$ Pour une étude plus précise du rapport entre le geste sous-tendu par l'intentionnalité et les arts performatifs, je me permets de renvoyer le lecteur à Lucia Angelino (dir.), Quand le geste fait sens, Paris, Éditions Mimésis, 2015.
} 
façon la plus exemplaire, dans le cinéma ${ }^{1}$. Il y voit — et à juste titre - un nouveau tournant tout aussi radical. Là même où le geste dé-fonctionnalise comme symptôme, s'ouvre un nouvel espace-temps, plus virtuel, plus paradigmatique de reconquête, au sein duquel l'humanité cherche à évoquer, voire à réveiller, « ce qui achevait de lui échapper à jamais $\|^{2}$. Dans cet autre espace - celui du cinéma et des arts en général - le geste survient à nouveau soit comme acte de l'image, soit comme acte du langage (autrement dit comme acte de parole): acte par lequel le corps "s'implique» intensément, c'est-à-dire se compose avec lui-même en une figure et "se déplie fabuleusement hors de lui même $»^{3}$, se faisant trace visible et durable de la motion vécue du sentiment qui s'exprime en lui. Du même coup, le geste, avec le pathos $^{4}$, dont il est indissociable, ouvre dans l'image ou dans le langage la sphère de l'éthos, autrement dit la sphère d'une " médialité pure et sans fin, qui se communique aux hommes $\rangle^{5}$, d'une expression radicale qui expose l'homme en tant que tel dans son " propre être-moyen $»^{6}$.

En d'autres termes, les gestes à l'œuvre ${ }^{7}$ dans les arts de la scène (ou de performance) - théâtre, danse, musique - mais aussi les gestes mis en images dans les arts de la trace - peinture et cinéma en particulier - nous confrontent à une expérience de l'expression tout à fait singulière ${ }^{8}$. Comme Maurice Merleau-Ponty s'en était très vite aperçu, ici l'esprit se fait corps, directement visible, dans ses gestes, ses attitudes et ses postures. Ici, « ce qui est au-dedans est aussi au-dehors ", selon l'expression de Goethe ${ }^{9}$, autrement dit, l'être humain est visible tout entier dans son corps et cette apparence

${ }^{1} C f$. Giorgio Agamben, « Notes sur le geste », dans Id., Moyens sans fins, op. cit., p. 64.

${ }^{2}$ Idem.

${ }^{3}$ Selon la belle formule que nous empruntons à Georges Didi-Huberman, « Préface » à Bertrand Prevost, La Peinture en actes, Paris, Éditions Actes Sud, 2007, p. 14.

${ }^{4}$ Le pathos est ici compris, conformément à la définition qu'en donne Aristote dans la Poétique, comme état affectif suscité chez le spectateur par un message ou un comportement particulier.

${ }^{5}$ Giorgio Agamben, « Notes sur le geste », dans Id., Moyens sans fins, op. cit., p. 69.

${ }^{6}$ Idem.

7 Selon une expression que nous empruntons à Barbara Formis (dir.), Gestes à l'œuvre, Paris, Éditions De l'Incidence, 2008.

${ }^{8}$ Sur ce thème, je me permets de renvoyer à Lucia Angelino, "L'intérêt de la conception merleau-pontienne du corps comme expression », dans Le Cercle Herméneutique, $\mathrm{n}^{\circ}$ 22-23, « Nouvelles études de Daseinanalyse », 2014, p. 13-27.

${ }^{9}$ Cité par Maurice Merleau-Ponty dans Le Cinéma et la nouvelle psychologie, Paris, Éditions Gallimard, 2009, p. 24. 
nous donne à saisir une intériorité, voire une «manière spéciale d'être au monde, de traiter les choses et les autres, qui définit avec évidence chaque personne que nous connaissons $\rangle^{1}$. De ce fait, nous sommes confrontés à la révélation d'un sens naissant à même le corps, c'est-à-dire offert à même un corps gestuel qui se fait moins efficient et opératoire, qu'il ne se prête à une motion ou à une émotion qu'il accueille et qu'il communique aux autres.

En cela, le geste réalise dans l'art (et dans l'expression esthétique) la tâche la plus difficile : transformer notre corps phénoménal (ou vécu) en un corps parlant, capable d'incarner et de mettre en scène nos impulsions, possibilités, forces et émotions les plus profondes, en faire un événement expressif susceptible de nous apprendre de nouvelles formes d'expression et d'interaction avec les autres, d'induire même de nouvelles formes (normes) de vie et de nouvelles manières d'être au monde, de produire de nouvelles formes de comportement et de coexistence avec les autres ; et, enfin, de nous toucher, d'un toucher figuré qui est à même de réveiller les potentialités étouffées ou dormantes de notre corporéité, de nous réapprendre le langage souvent oublié des gestes et des mouvements, celui profond et primordial de la communication visuelle et charnelle entre âmes incarnées. C'est ainsi, du moins, que je voudrais étudier le rapport entre le geste et l'image, en tant qu'opérateur d'intensification, de précipitation qui travaille dans la mise en forme sociale ou artistique des comportements et dans la composition des rapports entre les corps.

Dès lors, ma question directrice sera plus la suivante : «Qu'est-ce que les images font aux gestes $»^{2}$ ? Ou bien plutôt, comment la mise en scène artistique intervient-elle dans l'intensification de l'expressivité gestuelle et corporelle ? Et, au préalable, comment comprendre que des gestes mis en images puissent nous émouvoir et même mouvoir le présent de nos propres gestes, nous faire vivre et même ressentir notre corporéité vivante et vécue ? Comment comprendre qu'ils puissent éveiller un écho dans le corps du spectateur qui les reçoit et réveiller les expériences qui l'enracineront dans son esprit ? Pour répondre à ces questions, je chercherai avant tout à m'interroger sur la signification des gestes et sur l'efficacité de leur expressivité. Une telle interrogation sera articulée en trois étapes. Il nous faudra : (1) nous libérer de l'idée courante selon laquelle les gestes sont des mouvements n'ayant de sens que par rapport à une fin, à un résultat, à un objectif pragmatique, autrement dit, des moyens subordonnés à des fins pratiques, à des

${ }^{1}$ Ibid., p. 22-23.

${ }^{2}$ Pour un point de vue intéressant sur cette question et sur le rapport théorique gesteimage, voir Bertrand Prevost, La Peinture en actes, op. cit., p. 81 et suivantes. 
buts; (2) retrouver le noyau essentiel de la conception du geste selon Merleau-Ponty, conception suivant laquelle le geste est une expression, au sens que le philosophe français donnait à ce terme, à savoir une forme, au sens d'une Gestalt, en laquelle se réverbère la vie intérieure à laquelle notre corps est lié : un nœud des attitudes entrelacées les unes aux autres qui transforme notre corporéité en événement expressif et à ce titre opère une sorte de théâtralisation directe des énergies, puissances et forces du corps vécu ; (3) apprendre à voir que le geste, en tant que modalité, voire manifestation de la vie, a le caractère d'un se-montrer ou d'un venir-auparaître qui ouvre " la sphère non pas d'une fin en soi mais d'une médialité pure et sans fin qui se communique aux hommes $»^{1}$.

Conformément aux trois exigences énoncées jusqu'ici, trois temps scanderont mon parcours. Dans un premier temps, je me propose d'étudier le mouvement de la danse en tant que pur geste chorégraphique dépourvu d'une finalité pratique ou pragmatique, d'un « où-vers-où » au sens d'une prestation, qui réalise l'accord parfait de l'apparence et de l'essence, de l'âme et du corps, leur entrelacs. Ensuite, ce seront les gestes représentés en peinture et dans l'art du cinéma, que je vais considérer de façon plus approfondie. L'enjeu est au moins double. Il s'agit, d'une part, d'étudier la manière dont les gestes peuvent façonner et élargir notre sensibilité corporelle et, d'autre part, de mesurer l'impact éducatif ou le degré d'intervention qu'ils peuvent avoir dans l'invention de nouvelles formes de sentir et de réciprocité entre individus.

\section{Penser les gestes pour eux-mêmes : du mouvement de la danse aux gestes en peinture}

Pour approfondir la signification et la spécificité des gestes ${ }^{1}$, je vais d'abord essayer de les penser pour eux-mêmes. Penser les gestes pour eux-mêmes signifie pour l'essentiel penser des attitudes, des conduites, des manières d'être qui expriment au-dehors notre être intime et la nature profonde, affective et émotionnelle du lien psychique qui nous unit au monde et aux autres. Autrement dit, il s'agit de penser les gestes comme ensemble des mouvements et des conduites qui transfigurent notre corps, instaurant une théâtralisation de la vie intérieure à laquelle il est lié. Pour ce faire, je vais mettre en œuvre une sorte de suspension - de «mise en suspens » - de

${ }^{1}$ Selon une expression que nous empruntons à Giorgio Agamben, « Notes sur le geste », dans Id., Moyens sans fins, op. cit., p. 69. 
notre attitude naturelle qui nous porte à penser spontanément les gestes comme des mouvements n'ayant de sens que par rapport à une fin, à un résultat, à un objectif pragmatique.

Pour déconstruire cette manière habituelle et trompeuse de penser le geste, pour libérer les gestes du régime des fins, je propose de porter notre attention non pas tant vers le résultat auquel ils sont subordonnés comme moyens, mais plutôt vers leur manière d'être, leur forme - au sens d'une Gestalt - en laquelle se réverbère (s'exprime) notre vie intime et la nature profonde du lien psychique qui nous unit au monde et aux autres. Dans cette perspective, je partirai d'une analyse détaillée du mouvement de la danse qui, selon la subtile distinction qu'Erwin Straus permet d'opérer, est un " mouvement présentiel et non finalisé $»^{2}$, un mouvement non dirigé et non limité qui s'accomplit pour lui-même et non en vue de quelque chose d'autre, un mouvement auto-présentatif qui élargit le corps dans l'espace et s'accomplit dans un vivre participatif et phatique avec le monde; un pur geste chorégraphique dans lequel la tension entre sujet et objet, moi et monde, se

${ }^{1}$ Qu'est-ce qu'un geste ? C'est un mouvement, ou plutôt un acte où le corps « s'implique » intensément, c'est-à-dire se compose avec lui-même en une figure et « se déplie fabuleusement hors de lui même, se faisant trace visible et durable de la motion vécue du sentiment (ou de la perception) qui s'exprime en lui. Autrement dit, ce qui caractérise le geste c'est qu'il n'est pas question en lui «ni de produire ni d'agir» (Giorgio Agamben, "Notes sur le geste », dans Id., Moyens sans fins, op. cit.,. p. 68), mais d'accueillir et affronter, voire gérer (selon l'étymologie du terme provenant du Latin gestus, participe passé du verbe gerere qui signifie accomplir et porter, gérer) la révélation d'un sens qui affleure « à même un corps qui se fait moins actif, efficient ou opératoire qu'il ne se prête à une motion - et à une émotion qu'il accueille » et qu'il communique aux autres (cf. Jean-Luc Nancy, Le plaisir au dessin, Paris, Éditions Galilée, 2009, p. 50). Le geste est en ce sens, une expression, au sens que Merleau-Ponty donnait à ce terme, c'est-à-dire une transfiguration du corps, une gestalt ou une forme, en laquelle se réverbère la vie intérieure à laquelle il est lié, un nœud des attitudes entrelacées les unes aux autres qui transforme notre corporéité en événement expressif et à ce titre opère une sorte de théâtralisation directe du corps. Par ses gestes, le corps se compose une histoire en propre, « se fait sa petite histoire » ou plutôt « se fait son petit théâtre », il met en œuvre une certaine forme de narrativité, une temporalité ; il compose même une musique historiale qui se jouerait en mode mineur, quand bien même ses gestes se composent en un agrégat de micro-événements.

${ }^{2}$ Erwin Straus, «Les formes du spatial. Leur signification pour la motricité et la perception », dans Jean-François Courtine (dir.), Figures de la subjectivité. Approches phénoménologiques et psychiatriques, Paris, Éditions du Centre National de la Recherche Scientifique, 1992, p. 15 et suivantes. 
trouve pleinement suspendue, au profit de l'épreuve d'un devenir-un avec l'espace ambiant. En effet, dans ce mouvement, nous ne sommes plus tournés vers les objets singuliers du monde extérieur, "nous vivons notre présence, notre être vivant, notre sensibilité », ou plutôt une pure mobilité non dirigée qui vibre à l'unisson avec le mouvement propre de l'espace acoustique par lequel elle est induite de manière phatique ${ }^{1}$. C'est pourquoi les mouvements de la danse nous communiquent une exubérance ludique, une belle gratuité qui n'est pourtant pas dépourvue de sens $^{2}$, puisqu'elle consiste toute entière à exhiber le caractère médial, voire expressif des mouvements corporels. En reprenant les analyses de J. Patočka, nous pourrions dire en effet que les mouvements dansés, quoique «dépourvus d'une finalité pratique, d'un où-vers-où au sens d'une prestation selon le principe du maximum et du minimum », ont bien « un commencement et une fin spatiaux et un commencement et une fin purement temporels, ceux-là extérieurement limités, déterminés $d u$ dehors, ceux-ci intérieurement limités, déterminés $d u$ dedans $»^{3}$. En ce sens, ces gestes font sens ${ }^{4}$, aussi bien pour celui qui les exécute que pour celui qui les regarde, dans une dimension vécue, certes impalpable, mais fondamentale: leur sens n'est pas donné objectivement, mais intérieurement, comme l'équilibre et le rythme d'une mélodie.

Néanmoins, cette analyse serait loin d'être suffisante pour comprendre tout à fait la consistance esthétique et l'efficacité des gestes en tant que

1 À bien des égards, la danse d'Isadora Duncan aura décidément constitué la première émergence nette d'une danse faite pour fonctionner en ce sens, et toute entière tendue vers cet idéal. Selon ces propres termes, retrouver la beauté (le rythme naturel), perdue depuis des siècles, d'un mouvement naturel qui s'harmonise et concorde avec celui des vagues de la mer, des nuages dans le ciel, des arbres ondoyantes soumis aux caprices du vent, tel fût le problème auquel elle s'était vouée et pour lequel elle travailla. $C f$. en particulier Isadora Duncan, Ecrits sur la danse et $L a$ danse de l'avenir, Paris, Éditions Complexe, 2003, p. 53-71.

${ }^{2}$ Autrement dit, les mouvements de la danse sont dirigés non pas d'un point A vers un point $\mathrm{B}$, mais d'un ici vers un là-bas. L'ici et le là-bas étant les dimensions originaires de ce que E. Straus appelle l'espace du paysage, l'espace à même le sentir d'une présence au monde. En ce sens, on peut dire qu'il y a une précision dans le mouvement dansant qui n'est pas donnée objectivement, ni ordonnée à une fin, mais donnée intérieurement, de même que l'équilibre d'une mélodie.

${ }^{3}$ Cf. Jan Patočka, Papiers phénoménologiques, Grenoble, Éditions Millon, 1995, p. 50 .

${ }^{4}$ Sur ce thème je me permets de renvoyer à Lucia Angelino (dir.) Quand le geste fait sens, op. cit., 2015 et plus particulièrement à l'« Introduction : Entre sentir et faire : le geste qui fait sens », p. 15-31, note 1. 
mouvements expressifs. Puisque c'est précisément cette dimension et cette consistance esthétique et communicative des gestes qui m'intéresse, l'efficacité de leur expressivité, ce sont les gestes en peinture que je vais considérer de façon plus approfondie. La question se posera alors de savoir comment la peinture, les arts visuels en général peuvent nous faire voir, jusque dans l'immobilité de l'image, la mesure et la grâce des mouvements, nous suggérer même les émotions, les motions vécues du sentiment qui s'exprime en eux : comment la peinture - «poésie muette », muta poesia, selon le mot de Léonard - peut-elle rendre présente l'éloquence des gestes et nous faire éprouver même les affects auxquels ils sont liés ?

Dans un bref passage du Langage indirect et les voix du silence, Merleau-Ponty nous livre une ébauche de réponse - que l'on peut même considérer comme la clé de la solution : c'est par les mouvements des corps, qui s'y trouvent inscrits, que la peinture parle, en instaurant une éloquence des corps, corrélative d'une éloquence des gestes, envisagés comme «expression primordiale $»^{1}$, en proposant une théorie du geste et de l'expression capable de faire parler les corps.

Un bref aperçu de l'histoire des formes expressives, des procédés et des stratégies mises en œuvre par les artistes pour atteindre un tel résultat, semble d'ailleurs confirmer la justesse et la pertinence d'une telle observation.

Jusqu'à l'apparition du cinéma à la fin du XIX ${ }^{\mathrm{e}}$ siècle, la représentation du mouvement et de la gestualité humaine a pu se faire à un premier niveau à travers le langage des gestes, des mouvements et des attitudes, même les plus minimales du corps, amplifiées par les mouvements éphémères des êtres inanimés, comme les chevelures envolées, les vêtements, les drapés flottants. Tous ces mouvements et attitudes suspendues ne suggèrent pas seulement, sous un mode figé (arrêté), les mouvements réels, mais ont aussi pour but plus subtil de manifester les mouvements intérieurs auxquels ils sont liés, les états de l'âme, les vécus psychiques, les affects du personnage qui s'exprime en eux. Ce double enjeu narratif et expressif de l'âme et du corps est notamment au centre de la peinture de la Renaissance. Par une composition des mouvements, même minimaux, suggérés davantage qu'effectués, la

${ }^{1}$ Maurice Merleau-Ponty, «Le langage indirect et les voix du silence », dans $I d$., Signes (1960), Paris, Éditions Gallimard, 2001, p. 108 : « Tout usage du corps est déjà expression primordiale, — non pas ce travail dérivé qui substitue à l'exprimé des signes donnés par ailleurs avec leur sens et leur règle d'emploi, mais l'opération première qui d'abord constitue les signes en signes, fait habiter en eux l'exprimé par la seule éloquence de leur arrangement et de leur configuration ». 
peinture de la deuxième moitié du Quattrocento (de la Renaissance des années 1450-1460 à Florence) cherche à produire sur la toile une sorte de théâtralisation directe du corps : par des petits gestes - tel un doigt tordu, l'ondulation d'une chevelure - chaque personnage se compose une histoire en propre, « se fait sa petite histoire » ou plutôt «se fait son petit théâtre ${ }^{1}$, il met en œuvre une certaine forme de narrativité, une temporalité ${ }^{2}$.

Au-delà d'une représentation par les attitudes du corps, l'expressivité du mouvement et par conséquent de la vie intime, a pu être amplifiée et démontrée visuellement par d'autres moyens aux vertus cinétiques. C'est ainsi par exemple que Botticelli avait cherché un effet de souplesse en accentuant les mouvements des êtres inanimés — tels le battement d'une draperie sous la poussée du vent, l'ondulation d'une chevelure dans l'air, les vibrations d'un tissu, le déploiement des branchages, des feuillages, etc. Mais seul Léonard de Vinci trouva la vraie solution dans ce que les Italiens appellent le sfumato : un contour enveloppant, des couleurs adoucies permettant aux formes de se perdre les unes dans les autres et de nous communiquer, ainsi, l'imaginaire pulsation de la vie. C'est cette invention technique que Léonard employa dans la Joconde pour capter l'âme de son modèle et suggérer l'expressivité d'un visage qui se modifie en fonction de l'œil droit ou gauche que nous fixons. On pourrait d'ailleurs dire que la grâce qui anime son visage se trouve comme redoublée, mise en exergue par le regard direct qu'elle lance au spectateur. Le regard qu'elle pose sur nous est si aigu, si expressif, que l'on peine à croire que tant d'intensité suggérée n'est, après tout, qu'un peu de couleur sur une toile grossière. Ce qui nous frappe avant tout, c'est l'apparence de vie du personnage : « Comme un être vivant, elle semble presque changer devant nos yeux, et son visage nous apparaît presque différent chaque fois que nous y revenons $»^{3}$.

1 Bertrand Prévost \& Georges Didi-Huberman, La Peinture en actes : gestes et manières dans l'Italie de la Renaissance, op. cit., p. 192.

${ }^{2}$ Cf. Aby Warburg, La Naissance de Vénus et le printemps de Sandro Botticelli, Paris, Éditions Allia, 2007 : dans cet essai magistral sur les tableaux de Botticelli, A. Warburg a fait « de la peinture une question de geste (à travers la notion de Pathosformel) et du geste une question du temps (à travers la notion de Nachleben) », comme l'exprime de façon lumineuse Georges Didi-Huberman, dans la « Préface » à Bertrand Prevost, La Peinture en actes, op. cit., p. 14. Cf. Aby Warburg, Essais Florentins, Paris, Éditions Klincksieck, 2003 et Id., L'Atlas Mnémosyne, Paris, Éditions L'Écarquillé, 2012.

${ }^{3}$ Ernst H. Gombrich, L'Art et l'illusion, trad. fr. G. Durand, Paris, Phaidon, 2002, p. 227. 
Comme Léonard de Vinci et, plus encore que lui, Vélasquez a notamment exploité cette technique et cette réaction, en s'efforçant d'accentuer cette impression qu'il existe une "présence», "une forme de vie», un événement dans le tableau, qui compte sur notre participation (ou imagination) pour le suivre et suppléer ce qu'il a laissé ouvertement indéfini. L'exemple le plus significatif à cet égard se trouve dans le tableau Las Meninas d'où le regard d'un personnage s'adressant à nous sort du tableau pour y introduire notre propre regard. Un regard émanant du fond de la toile nous observe et nous amène ainsi à regarder à notre tour le tableau de manière plus attentive. Un tel dispositif - ce regard du peintre qui vient audevant du tableau vers le lieu d'où nous le voyons - attire notre attention et nous participons nous-mêmes à la scène. Au moment même où la figure peinte établit ce contact avec nos yeux, nous sommes contraints à nous attacher à ces formes et à retracer dans notre esprit le film de l'événement et, en nous attardant ainsi à cette représentation, nous en arrivons à éprouver nous-mêmes les sentiments des protagonistes représentés.

Nous voyons se dessiner ici un tournant décisif qui débouche sur l'art $\mathrm{du} \mathrm{XX}^{\mathrm{e}}$ siècle et ses rébus visuels lancés comme un défi à l'ingéniosité du spectateur. Autant dire que s'amorce ici un tournant décisif faisant évoluer de la « représentation linéaire » qui caractérise l'art archaïque, le « hiératisme » de l'art primitif, où la forme en tant que telle s'impose fortement au spectateur, à la «représentation picturale» qui trouva les moyens de susciter l'impression du mouvement - à travers la technique du sfumato, du non-fini introduite par Léonard de Vinci ${ }^{1}$. Au cours d'une telle évolution, le statut de l'image change radicalement. Elle n'est plus quelque chose d'immobile et d'autonome ; elle n'est plus un archétype. Elle est une invitation adressée au spectateur à la prolonger à son gré. En vertu des mouvements et des gestes qui s'y trouvent inscrits, elle se trouve chargée d'une énergie et d'une tension dynamique qui fait littéralement lever le potentiel de la sensibilité et de la mémoire du spectateur qui la reçoit, produisant une ouverture de son regard sur le tout dont elle fait partie. En ce sens, comme le remarque avec justesse Giorgio Agamben :

Même la Joconde, même Les Ménines peuvent être envisagées, non pas comme des formes immobiles et éternelles, mais comme des fragments d'un geste ou comme des photogrammes d'un film perdu, qui seul pourrait leur restituer leur véritable sens. Car toujours, en toute image, est à l'œuvre une

${ }^{1} C f$. à ce sujet notamment, le livre de Heinrich Wölfflin, Principes fondamentaux de l'histoire de l'art, trad. fr. C. Raymond, Brionne, Éditions Gérard Monfort, 1984. 
sorte de ligatio, un pouvoir paralysant qu'il faut exorciser ; et c'est comme si de toute l'histoire de l'art s'élevait un appel muet à rendre l'image à la liberté du geste $^{1}$.

Or, cela signifie que la rigidité de l'image s'est trouvée ici disloquée, et « qu'à proprement parler ce n'est plus d'images qu'il devrait être question ici, mais plutôt de gestes $»^{2}$, ou bien, selon l'heureuse expression introduite par Bergson et reprise par Deleuze, «d'images-mouvement $»^{3}$, d'images elles-mêmes en mouvement aptes à créer, ou à révéler, une multiplicités de formes diverses et à les composer entre elles dans un réseau qui les noue aux corps des spectateurs qui les regardent. Pourtant, quelle que soit la stratégie mise en œuvre, les gestes mis en images, représentés en peinture sont des gestes évoqués simplement à notre esprit, des gestes suspendus, suggérés davantage qu'effectués, des gestes rêvés, plutôt que réellement présents et agissants. «Introduire en ce rêve l'élément du réveil, telle » fût « la tâche du cinéaste $»^{4}$.

\section{Le cinéma : un nouvel art}

Comme Merleau-Ponty s'en était très vite aperçu, le cinéma représente en effet un nouvel art porteur de possibilités d'expressions nouvelles. Ici, l'esprit se fait corps, directement visible, dans ses gestes, ses attitudes et ses

\footnotetext{
${ }^{1}$ Giorgio Agamben, « Notes sur le geste », dans Id., Moyens sans fins, op. cit., p. 66.

${ }^{2}$ Ibid., p. 65-66.

${ }^{3}$ Sur ce concept d'image-mouvement que H. Bergson introduit dans le premier chapitre de Matière et Mémoire, avec ses trois formes principales - image-perception, image-action, image-affection —, je renvoie aux analyses lumineuses qu'y consacre Gilles Deleuze dans le premier volume de son livre sur Le Cinéma, intitulé, L'Imagemouvement, Paris, Éditions de Minuit, 1983 et plus particulièrement p. 7-22.

${ }^{4}$ Ibid., p. 67. Je pourrais prolonger ici l'analyse célèbre que Gilles Deleuze consacre à l'« image-mouvement », c'est-à-dire à l'image telle qu'elle apparaît au cinéma, comme un ensemble a-centré d'éléments variables qui agissent et réagissent les uns sur les autres et montrer qu'elle concerne, de façon générale, le statut de l'image dans la modernité. Toutefois, puisque mon objectif est de confronter l'expérience des gestes perçu au cinéma à la vision des gestes en peinture, analysée jusqu'ici, ce sont plutôt les analyses de Maurice Merleau-Ponty qui vont retenir mon attention, précisément parce qu'il s'intéresse au cinéma pour le confronter aux conditions générales de la perception et du comportement.
} 
postures. Ici, « ce qui est au-dedans est aussi au-dehors ${ }^{1}$, selon l'expression de Goethe, autrement dit l'être humain est visible tout entier dans son corps. Et cette apparence nous donne à saisir une intériorité. Ainsi comme le souligne Merleau-Ponty :

Colère, honte, haine, amour ne sont pas des réalités intérieures, accessibles à un seul témoin, celui qui les éprouve, ils ne sont pas des faits psychiques cachés au plus profond de la conscience d'autrui, ce sont des types de comportement ou des styles de conduite visibles du dehors. Ils sont sur ce visage ou dans ces gestes et non pas cachés derrière eux ${ }^{2}$.

Si donc le cinéma est destiné à devenir un art spécifique doté d'une esthétique propre, c'est que son texte, le texte du film réside en ce discours serré d'images et de sons, où chaque éclairage, chaque geste, chaque perspective a pour fonction de «faire paraître l'union de l'esprit et du corps, de l'esprit et du monde et l'expression de l'un dans l'autre $»^{3}$, de faire voir « le lien du sujet et du monde, du sujet et des autres » ${ }^{4}$. Ce qui fait l'art d'un film, c'est précisément la force et la subtilité de l'effet produit par l'arrangement temporel ou spatial de ses éléments. Comme le souligne avec force Merleau-Ponty :

Le sens du film est incorporé à son rythme comme le sens d'un geste est immédiatement lisible dans le geste, et le film ne veut rien dire que lui-même. L'idée est ici rendue à l'état naissant, elle émerge de la structure temporelle du film, comme dans un tableau de la coexistence de ses parties [...] Un film signifie comme nous avons vu plus haut qu'une chose [perçue] signifie : l'un et l'autre ne parlent pas à un entendement séparé, mais s'adressent à notre pouvoir de déchiffrer tacitement le monde ou les hommes et de coexister avec eux ${ }^{5}$.

En d'autres termes, dans la vie, comme dans l'art, le sens des gestes et des actions «ne se pense pas, il se perçoit $»^{6}$, et c'est là une expérience vécue tout à fait particulière, l'intérieur est à l'extérieur ou, si l'on préfère, à la surface.

${ }^{1}$ Maurice Merleau-Ponty, Le Cinéma et la nouvelle psychologie, Paris, Éditions Gallimard, 2009, p. 24.

${ }^{2}$ Ibid., p. 14.

${ }^{3}$ Ibid., p. 23.

${ }^{4}$ Idem.

${ }_{6}^{5}$ Ibid., p. 22 (je souligne).

${ }^{6}$ Idem. 
Toutefois, et c'est là une différence de principe avec la peinture et les autres arts visuels, le cinéma est un art du montage, du mouvement dans le temps et de la continuité organique. Ce que le film réalise par ce biais (cette invention technique), c'est une continuité visuelle sans faille dans la représentation des moments successifs d'une expression ou d'un événement. Le legato de la continuité visuelle, obtenu grâce à la technique du montage, nous donne à voir la polyphonie expressive d'un visage, par exemple, comme tout à l'heure le sfumato de Léonard de Vinci. Ce visage, nous le voyons changer, passer successivement par tous les stades intermédiaires des différentes tonalités affectives. Nous voyons chaque trait du visage (en particulier chaque trait de la bouche et des yeux) se détacher, l'un après l'autre, se détendre et se transformer lentement. Des minutes durant, nous suivons le processus organique (physionomique/mimique) de l'évolution des sentiments. Telle est bien la spécificité, la force expressive propre du cinéma qui en fait une description narrative des affects. Tandis que la technique picturale du sfumato, du non-fini laisse quelque chose à deviner, ne peut que renvoyer à une présence qui est au-delà et qui transparaît seulement; dans le legato de la continuité visuelle (obtenu grâce à la technique du montage), l'expression de l'instant présent porte encore la marque du précédent, et le suivant s'y dessine déjà ; nous ne voyons pas seulement les différents états d'âme du personnage, mais le processus mystérieux de la succession elle-même.

C'est pourquoi le cinéma apporte quelque chose de plus par rapport à la peinture, quelque chose de tout à fait particulier à travers ce caractère narratif des expressions et des conduites, des gestes et des sentiments. Il nous apporte la polyphonie expressive d'un visage où les contenus et les affects les plus variés peuvent apparaître simultanément, comme dans un accord musical, les relations entre les différents sons (notes) produisent un tout nouveau et irréductible aux éléments qui entrent dans la composition. D'une façon générale, la force expressive du cinéma (montage) réside en premier lieu en ceci qu'il nous donne à voir le rythme original, la transition même de nos émotions (et perceptions), d'une manière aussi impérieuse et saisissante que les gestes dont nous sommes témoins dans la vie. Il nous donne à voir tous les accords affectifs, dont la nature même et la consistance résident dans la simultanéité. C'est précisément là que réside le premier enrichissement apporté par le cinéma. Encore faut-il souligner que la force expressive du cinéma (i.e. du montage) nous fait «sentir» aussi «la coexistence, la simultanéité des vies dans le même monde », «les acteurs pour nous et pour 
eux-mêmes ${ }^{1}{ }^{1}$, comme tout à l'heure la Joconde et son regard liaient le spectateur et son regard à sa vie intime.

L'autre enrichissement considérable qu'apporte le cinéma réside dans le pouvoir d'exploiter le mouvement en tant que moyen d'expression. Au cinéma, le mouvement peut devenir la plus intense expression d'un rythme de vie ou d'un sentiment. Par exemple, une allure de plus en plus rapide devient l'expression d'une impatience croissante, de même qu' " un corps déséquilibré qui se tord sur un rocher » ou " cette marche vacillante qui tente de s'adapter à on ne sait quel bouleversement de l'espace $»^{2}$ nous donne le sentiment du vertige. Nous éprouvons véritablement dans notre propre corps le vertige en le voyant ainsi de l'extérieur, en contemplant, tel que le cinéma nous permet de le faire, " ce corps déséquilibré qui se tord sur un rocher ${ }^{3}$. D'une façon générale, si au cinéma les mouvements ont tant d'intensité, c'est parce qu'ils sont des spectacles du corps humain. Nous pourrions dire que, grâce au premier plan qui met en relief la physionomie et les mimiques, le cinéma dote les gestes d'une évidence et d'une efficacité extraordinaires : ceux-ci non seulement offrent à notre vie intérieure (à nos sentiments) une forme en laquelle elle peut s'exprimer, mais peuvent aussi, en retour, l'induire, la façonner et la nourrir, la maintenir en mouvement: autant un sentiment peut se traduire immédiatement dans un geste, autant un geste peut provoquer un sentiment.

\section{Force et faiblesse du cinéma}

Nous pourrions en conclure que le cinéma - né de la grande industrie du capitalisme - est en train de faire prendre à la civilisation un nouveau tournant tout aussi radical, en ceci que, à travers lui, l'humanité est en train de réapprendre le langage souvent oublié des gestes, des mimiques, et des mouvements, celui profond et primordial de la communication visuelle et charnelle entre âmes incarnées. Comme s'en était aperçu très tôt MerleauPonty, ici, l'esprit se fait corps, directement visible, dans ses gestes, ses attitudes et ses postures. Ainsi, nous retrouvons cette expérience primordiale d'une communication et d'une compréhension immédiate, spontanée, profonde, émotionnelle avec les autres, que les gestes nous font éprouver

\footnotetext{
${ }^{1}$ Ibid., p. 18

${ }^{2}$ Ibid., p. 23.

${ }^{3}$ Idem.
} 
originellement et qui s'obtient — sans médiation interposée — «par la réciprocité de mes intentions et des gestes d'autrui »" à chaque fois que «les pouvoirs de mon corps s'ajustent » aux intentions « lisibles dans la conduite d'autrui $»^{2}$. C'est bien de cette expérience primordiale que nous commençons à nous souvenir, c'est bien encore ce langage des mouvements, des gestes que nous sommes en train de réapprendre. C'est le cinéma, comme nous l'avons vu, qui fait réapparaître ce langage natif, enseveli sous les mots usés, les conventions et les idées reçues - ainsi que le remarque Merleau-Ponty :

Le cinéma ne nous donne pas, comme le roman l'a fait longtemps, les pensées de l'homme, il nous donne sa conduite ou son comportement, il nous offre directement cette manière spéciale d'être au monde, de traiter les choses et les autres, qui est pour nous visible dans les gestes, le regard, la mimique, et qui définit avec évidence chaque personne que nous connaissons ${ }^{3}$.

Nous trouvons donc confirmée l'idée que le cinéma marque un tournant décisif et nouveau dans notre société post-industrielle, qui peut favoriser le passage d'une civilisation intellectualisée à l'excès et devenue abstraite à une civilisation visuelle, animée par le désir, voire par l'aspiration humaine à récupérer l'expérience vécue d'une réalité concrète, non médiatisée, celle encore d'une communication immédiate et spontanée avec les autres.

Ce chemin peut pourtant mener dans deux directions apparemment opposées. D'une part, le cinéma peut apprendre aux hommes de nouvelles possibilités gestuelles ainsi que des formes d'expression nouvelles, favoriser le passage d'un savoir rationnel et sclérosé au réveil d'une sensibilité corporelle irréfléchie, riche en possibilités communicatives et susceptible de féconder notre vie affective et notre imagination ${ }^{4}$. D'autre part, ce même

${ }^{1}$ Maurice Merleau-Ponty, Phénoménologie de la perception (1945), Paris, Éditions Gallimard, 2004, p. 215.

${ }^{2}$ Idem.

${ }^{3}$ Maurice Merleau-Ponty, Le Cinéma et la nouvelle psychologie, op.cit., p. 23.

${ }^{4}$ À ce titre, il faut remarquer en premier lieu que le cinéma peut nous remettre au contact avec l'expérience vécue, immédiate, concrète, non conceptuelle des choses, avec la riche physionomie vivante qu'elles ont toutes et qui a été éclipsée par le processus de réification inscrit dans la nature même du capitalisme. En second lieu, à travers le jeu de la physionomie et des mimiques, il peut nous réapprendre le langage des affects, des gestes qui dénote avec intensité, des événements intérieurs, nous apprendre à déceler le rythme originaire des sentiments dans l'expression d'un visage. En troisième lieu, à travers la technique du «gros plan», l'art de l'accentuation, il peut attirer l'attention sur ce qui compte et fait sens dans une histoire, nous montrer par exemple le moment décisif d'une action «motivée » (et non mécanique), 
chemin, qui semble promettre une libération de notre potentiel humain expressif et créateur, peut nous délivrer une autre malédiction, celle de la soumission à des messages « subliminaux » qui, s'adressant à une sphère non rationnelle, celle de l'émotivité, de la sensibilité inconsciente, échappent à notre contrôle (au niveau préréflexif ou préconscient de la conscience) et peuvent façonner notre vie, à notre insu.

Il faut reconnaître en effet que, avant même de fonctionner comme des « discours muets », les gestes humains - mais aussi bien les images qu'ils composent avec nos corps pour véhicule — sont affaire d'attraction et de répulsion, et avant tout d'identification. Autant dire que le cinéma, comme tous les arts figuratifs, sitôt qu'ils mettent en scène des corps, dote les gestes d'une évidence, leur confère une visibilité, mais cette visibilité est en ellemême aveugle; elle s'opère de façon irréfléchie, presque naturellement, automatiquement. Elle est en soi problématique, puisque sémantiquement équivoque. Le geste comme toute chose perçue, évoque énigmatiquement un sens, une présence, il émet un message non intégralement déchiffrable. Il peut seulement fournir un indice, alimenter la sensation d'une présence, sans fournir aucune démonstration. Il est autant ambigu et fugitif que l'est la ligne de vol d'un oiseau. En un mot, il nous fait signe, mais n'est pas un vrai signe. Pour marquer cette subtile distinction, on peut convoquer ici le concept husserlien de l'Anzeichen, du signe motivationnel indicatif qui renvoie à d'autres objets ou à d'autres états, quoique sans pouvoir en démontrer l'existence, comme le fait en revanche l'Ausdrücke, le signe expressif ${ }^{1}$.

le motif, au sens de ce qui la motive, l'origine, et la conséquence d'un acte, sa naissance et sa métamorphose.

${ }^{1}$ Cette distinction constitue le centre de la théorie husserlienne du signe élaborée dans les Recherches Logiques et nous amène à la critique de Jacques Derrida formulée dans La voix et le phénomène. Introduction au problème du signe dans la phénoménologie de Husserl, Paris, PUF, 1967. En effet, bien que le mot signe ait un double sens - d'indice (signe distinctif, marque, etc.) et d'expression - chez Husserl dès le début de la Recherche 1 (§ 1-16), Husserl établit clairement que le signe indicatif motivationnel (l'indication) ne peut pas fonder une signification idéale, mais seulement une relation associative entre ses éléments (le signe et l'objet), tandis que la signification repose uniquement sur un acte intentionnel donateur de sens, jamais sur une association contingente. En ce sens, le signe indicatif dont parle Husserl n'est pas un vrai signe ; il ne se substitue pas aux choses mais renvoie à elles; il n'est pas mis à la place de quelque chose, mais renvoie à quelque chose. C'est un certain rapport motivationnel, associatif, occasionnel que l'on ne peut réduire à un quelconque acte actif de donation de sens, mais seulement à une sorte de motivation associative-indicative, qui, à son tour présuppose une 
L'Anzeichen (signe indicatif) renvoie à, indique, évoque, suggère énigmatiquement un sens, une présence, un sentiment, un état de l'âme, une manière d'être ; il nous pousse même obscurément à y croire sans pourtant nous en fournir aucune démonstration. Il déclenche ainsi un processus de renvoi sans fin où chaque indice finit par renvoyer à son tour à quelque chose d'autre. Mais les renvois analogiques que produisent les gestes sont confus, éphémères et inquiétants, ambigus et multiples. Ainsi, on n'oserait plus guère soutenir aujourd'hui que les passions et les événements puissent être exprimés directement, se refléter entièrement dans des gestes, comme c'était le cas à l'époque où était en vogue le théâtre. Les gestes parlent, certainement; mais si incomparable et impérieuse que puisse être quelquefois l'expression de l'indicible, de l'ineffable, du sentiment dans le geste ou dans l'image, elle ne s'en produit pas moins de manière plus intense que dans la proximité immédiate du mot qui l'accompagne, comme une sorte d'aura autour de son sens insondable. Les gestes parlent seulement s'ils sont vécus dans la proximité et dans la ténacité silencieuse d'une parole qui les affronte et s'y confronte pour les faire sortir de leur silence. C'est pourquoi, en dépit de son immense popularité, le cinéma ne pourra pas, à lui seul, transformer notre société en quête d'une communication immédiate et spontanée. Il ne pourra atteindre ce résultat que si l'évolution générale du monde ambiant crée une atmosphère culturelle en affinité avec lui ${ }^{1}$.

implication intentionnelle cachée, une ambiance ou une audience communes que Husserl appelle un "horizon de situation》 dans la Logique formelle et logique transcendantale, trad. fr. S. Bachelard, Paris, PUF, 1957. Pour ce qui concerne la théorie husserlienne de la signification et le double sens du terme signe, voire Edmund Husserl, Recherches logiques (1961), trad. fr. H. Élie et alii, Paris, PUF, 2011 et en particulier Recherche 1. «Expression et signification », chapitre 1 «Les distinctions essentielles» (§1-16). Pour revenir à la critique de Derrida formulée dans La Voix et le phénomène, elle s'attaque précisément à l'approche logique de la signification chez Husserl, autrement dit au postulat d'une primauté de la signification idéale sur les autres facteurs du langage. Ce cadre étant rappelé, il s'agit de remarquer que le corps gestuel et expressif défie cette distinction en ceci précisément qu'il nous offre une "signifiance immanente, sans sortie du signe vers le signifié, [...] un sens offert à même le corps, à même un corps qui se fait moins actif, efficient, ou opératoire qu'il ne se prête à une motion - et à une émotion - qu'il accueille » et qu'il exhibe. $C f$. Jean-Luc Nancy, Le Plaisir du dessin, Paris, Éditions Galilée, 2009, p. 50.

${ }^{1}$ Pour approfondir ce thème, je renvoie au livre capital de Bela Balázs, L'Être visible et l'esprit du cinéma, Belval, Éditions Circé, 2010. 
En ce sens, je suis tentée de suggérer que le seul véritable geste, propre à nous apprendre des nouvelles formes d'expression et d'interaction avec les autres, d'induire de nouvelles normes de vie et de nouvelles manières d'être au monde, de produire de nouvelles formes de comportement et de coexistence avec les autres, n'est pas tant celui que le cinéma - et les arts en général - introduisent dans l'image, mais bien plutôt celui que l'écrivain introduit dans le langage : finalement c'est le geste de l'écriture, ou, bien plutôt, de la parole, que produisent la philosophie ou la littérature. En écrivant, on accomplit en effet un geste (intérieur) qui peut non seulement transformer notre vie intime, mais s'insinuer charnellement dans les pensées de l'autre, induire des sentiments, "émouvoir, bouleverser, récréer, surprendre l'homme, lui faire flairer de nobles pensées ou, en un mot, lui faire vraiment "vivre" quelque chose, être une "expérience vécue", comme le dit Robert Musil.

Encore faut-il remarquer, que ce pouvoir n'est effectif que dans ce que Merleau-Ponty appelle une parole parlante, authentique, « originaire $»^{2}$, celle qui formule pour la première fois ce qui est pensé ou perçu et qui, à la différence de la parole parlée - laquelle se contente de traduire une pensée déjà faite et «jouit des significations disponibles comme d'une fortune acquise $»^{3}$ est créatrice d'un sens nouveau, comme la parole « de l'enfant qui prononce son premier mot, de l'amoureux qui découvre son sentiment», de l'écrivain, et du philosophe qui « réveillent l'expérience primordiale en deçà des traditions ${ }^{4}$. À cet égard, la parole parlante ressemble bien à un geste corporel dont on comprend immédiatement le sens, car loin de renvoyer à un vécu psychique qui serait caché derrière elle porte avec elle son sens, « comme un geste contient le sien $»^{5}$. C'est bien la corporéité de la parole ce que Merleau-Ponty appellera son "épaisseur sémantique » (F. Ponge) ou encore son « humus signifiant» (J.-P. Sartre) - qui fait sens. Son apparence visuelle et sonore, qui n'en livre souvent qu'un aspect incomplet et brouillé, son expressivité, sa richesse sémantique, constituent autant d'éléments par lesquels elle est par elle-même et initialement signifiante, sans référence à une signification qui existerait en $\operatorname{soi}^{6}$. D'où l'idée que la parole soit origi-

\footnotetext{
${ }^{1}$ Robert Musil, L'Homme sans qualités, trad. fr. P. Jaccottet, tome 2, Paris, Éditions du Seuil, 2004, p. 204.

${ }^{2}$ Maurice Merleau-Ponty, Phénoménologie de la perception, (1945) Paris, Éditions Gallimard, 2004, p. 207, note 1, p. 208.

${ }^{3}$ Ibid., p. 229.

${ }^{4}$ Ibid. p. 208.

${ }^{5}$ Ibid., p. 214.

${ }^{6}$ Ibid., p. 446.
} 
nellement quelque chose «que l'on dit, que l'on entend et que l'on voit ${ }^{1}$, un événement qui vient au-devant de notre propre corps et se laisse ressaisir par lui à partir de l'expérience qu'il suscite en lui, un geste empreint d'une signification, immédiatement lisible dans la texture même de ses traits sensibles. Elle se donne elle-même à voir au lieu de disparaître dans ce qu'elle nous donne à voir et, à ce titre, peut nous émouvoir, nous surprendre, nous faire vraiment « vivre » quelque chose, être une « expérience vécue ».

\section{Conclusion}

Sans pouvoir m'engager davantage sur cette dernière remarque qui serait un trop long détour ici, je souhaiterais revenir à mon point de départ et m'expliquer brièvement sur le thème choisi pour cet essai : L'efficacité des gestes mis en images. Pourquoi partir de ce thème pour aborder la question de la modernité ? Une première réponse, assez simple, de laquelle nous sommes partis, consiste à dire que la perte des gestes expressifs, la catastrophe généralisée de la gestualité, constitue l'un des signes de la crise de la modernité. Partant de ce simple constat, il s'est agi de montrer que la gestualité, le mouvement libre, spontané, immédiat qui, d'après le toucher, constitue le trait distinctif et le plus essentiel de la corporéité humaine, continue cependant à s'exprimer dans le domaine de l'esthétique au double sens de l'aisthésis et de l'espace ouvré par les arts. De ce fait, en prenant pour thème les gestes mis en images nous avons voulu étudier la manière dont ils peuvent en retour ouvrir à nouveau les chemins de la liberté, façonner et élargir notre sensibilité corporelle, nous toucher, d'un toucher figuré qui est à même de réveiller les potentialités étouffées ou dormantes de notre corporéité, de nous apprendre ou plutôt de nous réapprendre le langage souvent oublié des gestes, des mimiques et des mouvements, celui profond et primordial de la communication visuelle et charnelle entre âmes incarnées. Il nous est apparu en effet que les images douées de mouvement s'éprouvent en quelque sorte du dedans et nous engagent dans une situation sensori-motrice qui change, ou plutôt intensifie non seulement notre perception, mais aussi notre sensibilité et notre disposition envers autrui. Ainsi, et de manière plus essentielle, si ce thème de la gestualité est susceptible d'intérêt, c'est parce qu'il préfigure et renouvelle en même temps les recherches actuelles sur

${ }^{1}$ Ibid., p. 273. 
l'empathie ${ }^{1}$. Il nous montre précisément que le mouvement, la gestualité, l'expressivité des corps est ce qu'il y a de plus intuitif et de plus profond dans la compréhension et la communication entre âmes incarnées, ce qui se situe à l'origine de toute forme d'empathie, indépendamment de la subtile distinction pouvant se faire entre le sentiment et la sensation, entre la connaissance et l'intuition ${ }^{2}$.

${ }^{1}$ Rien n'est plus complexe ni divise davantage les philosophes que l'interprétation de ce phénomène complexe qu'on appelle empathie et de l'acte qui la caractérise. L'empathie ou Einfühlung, provenant du verbe fühlen (sentir) nous confronte en effet à une modalité spécifique du sentir qui se qualifie pour le mouvement d'union ou d'identification avec un objet autre ou extérieur à soi qui, pourtant exprime quelque chose qui nous est intérieur, un sentiment ou un état de l'âme. Il s'agit d'un se sentir à distance de soi, dans un être extérieur et autre que soi. Plus exactement l'empathie désigne l'acte par lequel nous réalisons qu'autrui est sujet d'une expérience comme nous le sommes et qu'il est en train de vivre des émotions, ou des perceptions, d'accomplir des actes cognitifs ou volitifs que nous pouvons nous-mêmes comprendre et même partager. Mais qu'est-ce que cela signifie exactement? Et surtout comment comprendre que cela soit possible ? Comment interpréter précisément cet acte ? Comment différencier les actes d'empathie d'autres actes de la conscience? Le débat sur ce thème est plus que jamais vivant aujourd'hui. Pour un aperçu historique des principales doctrines de l'empathie, voire Andrea Pinotti, « Arcipelago empatia. Per un'introduzione", in Id. (dir.), Estetica ed empatia, Guerini, Milano, 1997; Id., «Empatia: un termine equivoco e molto equivocato », dans Discipline filosofiche, vol. $12, \mathrm{n}^{\circ} 2,2002$, p. $63-83$, où se trouve analysée en particulier la conception de l'empathie selon Theodor Lipps. Andrea Pinotti, Empatia. Storia di un'idea da Platone al post-umano, Bari, Laterza, 2011. À ce sujet voir également Dan Zahavi, «Beyond empathy. Phenomenological approach to intersubjectivity », dans Journal of Consciousness Studies, vol. 8, n 57, 2001, p. 151-167.

${ }^{2}$ Comme Theodor Lipps l'explicite de façon lumineuse dans l'article «Empatia e godimento estetico »: "L "empathie" est un terme ambigu (équivoque) et très mal compris, assez souvent malentendu. Cela est lié tout d'abord au fait que certains comprennent le "sentiment" [Gefühl] en un sens assez restreint comme sentiment de plaisir [Lust] ou de chagrin [Unlust], et qu'ils identifient le "sentir" [Fühlen] à l'épreuve du plaisir ou de déplaisir. Pour celui qui, de manière si illégitime, comprend le terme "sentiment" en un sens si étroit, l'"empathie", bien que concernant l'expérience du sentir, ne mérite pas cependant une telle appellation (nom). Car ce que j'empathise est, en sens absolument général, vie. Et vie est force, un intérieur opérer, aspirer et accomplir. En un mot, vie est activité ; [...] Plus profondément et radicalement on pourrait dire, en un sens figuré : "activité" est souffle intérieur ou pulsation intérieure ; ou, plus en général : c'est un mouvement intérieur, un mouvement vécu. Toutefois, le mouvement n'est pas à comprendre ici comme une simple impulsion en moi, mais il consiste dans le fait que je me meus. Bien évidemment, ce 
"mouvement" n'a rien à voir avec la spatialité. [...] Par exemple, j'empathise une vie vigoureuse et saine qui s'exprime dans la forme d'un corps humain ; par conséquent, j'appelle ce même corps énergique et sain. J'empathise une activité qui se déploie et se répand dans une ample salle. Dans un autre cas j'empathise la joie, la tristesse, le désespoir dans les gestes et dans les mots d'un être humain. Et tous ces vécus désignent une modalité de mon activité ou de l'activation de moi-même. [...] À cet égard, j'observe qu'il existe un autre terme qui semble indiquer le même phénomène désigné par le terme d'empathie : c'est le terme "expression". Un geste — comme on le dit souvent - exprime joie ou tristesse. Les formes d'un corps expriment force ou bien-être. Un paysage exprime une tonalité affective ou un état de l'âme [Stimmung]. Cet exprimer signifie en effet exactement ce que signifie le terme empathie " («Empatia e godimento estetico », dans Discipline filosofiche, vol. 12, $\mathrm{n}^{\circ} 2,2002$, p. 31-33, je traduis de l'italien au français). Pour poursuivre la discussion sur l'ambiguité qui entoure le concept d'empathie, voir le livre de Laura Boella, Sentire l'altro. Conoscere et praticare l'empatia, Milano, Raffaello Cortina Editore, 2006, p. XXX. 